\title{
Handover Operation in Mobile IP-over-MPLS Networks
}

\author{
Vasos Vassiliou \\ Department of Computer Science, University of Cyprus, \\ 75 Kallipoleos Str, 1678 Nicosia, Cyprus \\ vasosv@cs.ucy.ac.cy
}

\begin{abstract}
This paper examines mobility operations, and particularly handovers in an environment, where Hierarchical Mobile IP is operating over MPLS. The work in this paper improves on existing methods of MIP-MPLS interworking first by defining a simple framework based on Hierarchical Mobile IPv6 (HMIPv6) and second by outlining the relevant protocol design assumptions. In addition, the combined protocol is examined in detail under two scenarios and a comprehensive explanation of the operation of intra- and inter-cell handovers is presented.
\end{abstract}

\section{Introduction}

The evolution in mobile networks has given rise to several different yet complementary access networks such as second and third generation wireless cellular $(2 \mathrm{G} /$ $3 \mathrm{G}$ ), wireless local area networks (WLAN), and high altitude and satellite networks that offer a broad range of services targeted towards diverse subscriber needs. IPbased wireless networks are a research area of importance since the networks proposed for Universal Mobile Telecommunications System (UMTS) and the next generation $(4 \mathrm{G})$ of wireless networks are all-IP based.

To provide satisfactory services to the customers, handover delays, control messages and radio link inefficiencies need to be reduced. Current cell technologies cannot efficiently and cheaply support the density of infrastructure. Innovative interfaces and smaller cells are the solutions to these problems. Smaller cells mean increased signaling when legacy mobility protocols are used. However, in IP-based networks micro-mobility can easily be handled by hierarchical mobile IP (HMIP). The increased requirements of an IP-based radio access network (RAN) can be solved when the scalability and reduced latency of HMIP is combined with the switching performance and traffic engineering capabilities of multiprotocol label switching (MPLS).

The distinguishing feature of MPLS is the ability it offers to users to specify, and tightly control, the communication paths based not only on hop information but also a wide range of Quality of Service (QoS) parameters and policies. Given the tremendous increase in the use of wireless devices to access the Internet and multimedia services, concerns related to providing and maintaining specific service levels arise. It is therefore reasonable to consider an extension of MPLS into the mobile domain.

The goal of the research presented in this paper is to explain how mobility can be introduced, and especially how handovers can be handled, into a Micromobilityenable MPLS network using hierarchical mobile IPv6 (HMIPv6). We consider 
combining the two protocols in an overlay fashion for reasons of simplicity. This work builds on our previous work [5][6] which proposed and examined a framework for the integration of MPLS and HMIPv6 for use in a Radio Access Network.

\subsection{Background}

Multiprotocol Label Switching: MPLS [1] is a packet forwarding technology that assigns packet flows to label switched paths (LSPs). Packets are classified at the network edge based on forwarding equivalence classes (FECs). FECs summarize essential information about the packet such as destination, precedence, VPN membership, QoS information, and the route of the packet chosen by traffic engineering (TE). Based on the FEC, packets are labeled, and then transported over a label switched path based on that label. Packets belonging to the same FEC get similar treatment by all intermediate nodes in the path.

MPLS operates between layer two (data link) and layer three (network) of the protocol stack, thus it is referred to as a 2.5 layer architecture. To forward an unlabeled packet MPLS first relates the FEC with an entry in its next hop forwarding equivalence class table (NHLFE). This is done in the FEC-to-NHLFE (FTN) table. The NHLFE table contains the next hop, the operation to be performed on the packet (pop, push, swap) and a new label (if necessary). In a practical implementation the NHLFE also includes the incoming label of a packet so that it can handle labeled packets as well. The resulting table is called the label forwarding information base (LFIB) $)^{1}$.

Mobile IP: Mobile IP (MIP) allows a mobile node (MN) to move from one link to another without changing the mobile node's home IP address [2]. A home address is an IP address assigned to the mobile node within its home subnet prefix on its home link. Packets may be routed to the mobile node using this address regardless of the mobile node's current point of attachment to the Internet, and the mobile node may continue to communicate with other nodes (stationary or mobile) after moving to a new link. While a mobile node is attached to some foreign network, it is also addressable by one or more care-of addresses (CoA). When away from home, a mobile node registers one of its care-of addresses with a router on its home link; requesting this router to function as the home agent (HA) for the mobile node. The HA intercepts, encapsulates, and forwards packets to the mobile node through its registered $\mathrm{CoA}$.

Hierarchical Mobile IP: Hierarchical Mobile IP (HMIP) is a micro-mobility management model. Its purpose is to reduce the amount of signaling to correspondent nodes and the home agent and improve the handoff speed performance of mobile IP. HMIPv6 [3] is based on MIPv6 [4] and introduces a new entity called the mobility anchor point (MAP), and minor extensions to the mobile node and home agent operations. The major idea is that the mobile node registers the MAP's CoA with its home agent. Therefore, when the mobile node moves locally (i.e. its MAP does not change), it only needs to register its new location with its MAP. Nothing needs to be communicated with the home agent or any other correspondent nodes $(\mathrm{CN})$ outside

\footnotetext{
${ }^{1}$ NHLFE and LFIB are used interchangeably in this paper.
} 
the RAN. By using this method, signaling is contained in a smaller area, does not overwhelm the core network and the time to complete the location update is smaller.

The rest of the paper is organized a follows. Section 2 presents the design issues that need to be considered during the development of a framework for the interaction of MPLS and hierarchical mobile IP. Section 3 presents our proposed overlay HMIPv6-MPLS framework. Section 4 details the operation of intra- and inter-cell handovers in the resultant framework. Special attention is given to the data delivery processes. Section 5 summarizes the contributions of this work.

\section{Framework Design Considerations}

\subsection{Overview}

We distinguish between an overlay and an integrated framework of combining the operations of MPLS and HMIP at the level of interaction between the different architectures. In the overlay method, HMIP, and MPLS remain as separated as possible, without having any merged processes or signaling. Simple events or processes may then require additional messages or additional interaction between architectures to achieve the same result. Therefore, overlay frameworks usually introduce more latency and overhead. On the other hand, an integrated framework merges and relates many of the functions of its composing members. There exists a tradeoff between the simplicity of operation and the optimization and system performance. In this work we are focusing on the overlay paradigm. Our solution for the integrated paradigm can be found in [5] and [6].

\subsection{Radio Access Network}

The basic topology for this research work is a Radio Access Network (RAN) as shown in Fig. 1.

The RAN consists of at least three layers of label switched routers (LSRs). The edge components of the architecture are the radio access routers (RAS), which are the first IP aware devices of the network seen from the mobile terminal. One, or more, base stations (BS) are attached to a RAS (or integrated into it) and provide the physical radio link to the mobile node $(\mathrm{MN})$. Several RASs are interconnected to one or more Edge Gateways, which in turn provide access to outer (backbone) networks including other RANs. The RASs and the EGWs are linked through a network of MPLS-capable routers. We assume that all routers in the RAN can act as mobility agents (MA) to support mobility management based on hierarchical mobile IP.

The design of the reference network has been based on network architectures used by well-established providers and was also influenced by traffic engineering considerations as these are described in [7]. Enhancements to the network may include additional layers of hierarchy and more complex interconnection like full mesh or double homing.

Fig. 1 shows a general multi-RAN scenario where the mobile node traverses the center RAN. References will be made to nodes in this schematic in later sections. 


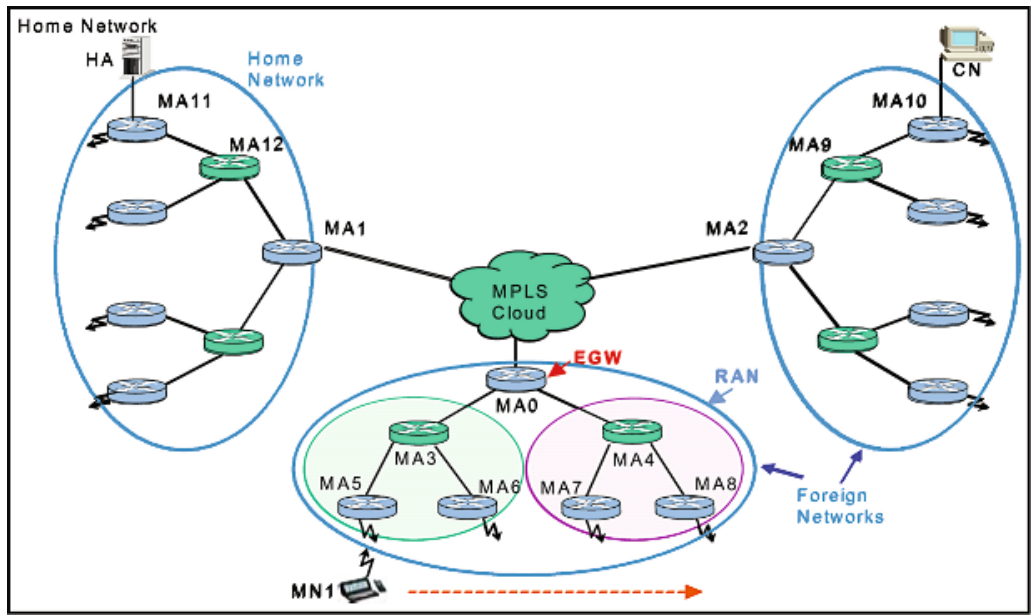

Fig. 1. Reference Network

\subsection{Design Assumptions and Specifications}

The design of the MIPv6 and HMIPv6 based MPLS framework is based on the following assumptions:

- All MPLS nodes in the RAN are mobility-enabled

- Mobile IP procedures for agent discovery, mobile node registration, and routing remain unchanged.

- Mobile nodes have no MPLS related protocols in their stack

- Only point-to-point LSPs are considered.

- MPLS operates in the following modes:

- $\quad$ Downstream on demand: An LSR explicitly requests a label binding for an FEC from its next hop for that particular FEC.

- Ordered control: An LSR only binds a label to a particular FEC if it is the egress for that FEC, or if it has already received a label binding for that FEC.

- Conservative retention: An LSR discards any label bindings from downstream routers if those routers are not its next hop (or no longer its next hop) for a particular FEC. This retention mode requires an LSR to maintain fewer labels.

- There is a unique label per LSP (i.e., there is no label merging). An LSR can support label merging if it has bound multiple incoming packets to an FEC that uses a single outgoing label. Once packets are transmitted using this method there is no way of differentiating them in terms of their source (input interface or incoming labels). Without label merging, if two packets for the same FEC arrive with different incoming labels they must be forwarded with different outgoing labels.

- No aggregation allowed (i.e., more than one LSPs for the same FEC is acceptable). Aggregation is the procedure of binding a single label to a union of FECs; it is itself an FEC (within a domain). 
- In our framework we would like to be able to have the finest granularity of label switched paths. For that reason we allow more than one LSPs for the same FEC from the same end node. FECs are defined on end-node pairs and QoS requirements.

- No penultimate hop popping is considered.

- The Data-driven method is used for the establishment of paths in a mobile network. An LSP is established only if data needs to be transferred between nodes.

We assume that the mobile node has already done address auto-configuration and registration, and has received an acknowledgement from its home agent. The CoA registered with the home agent is that of the mobility agent located at the edge gateway. Before we get into the details of the framework we have to consider other design issues.

\section{Binding Update - LSP Setup Relationship}

The relationship between the home agent binding update procedure and the home agent-mobility agent LSP setup can take many forms. Previous work has considered two methods for combining them: sequential (Fig. 2a) and encapsulated (Fig. 2b). In the sequential method the two procedures are initiated one after the other, with the LSP setup following a successful binding update (exchange of BU and BUAck). In the encapsulated method the home agent initiates the LSP setup after it receives the binding update message from the mobile node. However, a binding update acknowledgement message is not sent back until the LSP setup is complete.

- The sequential method of registering the MN to HA and establishing the related LSP is used. ${ }^{2}$

\section{Binding Update Acknowledgement}

Most of the current proposals use binding acknowledgement messages during Registration, even though they are not a requirement of the mobility protocol.

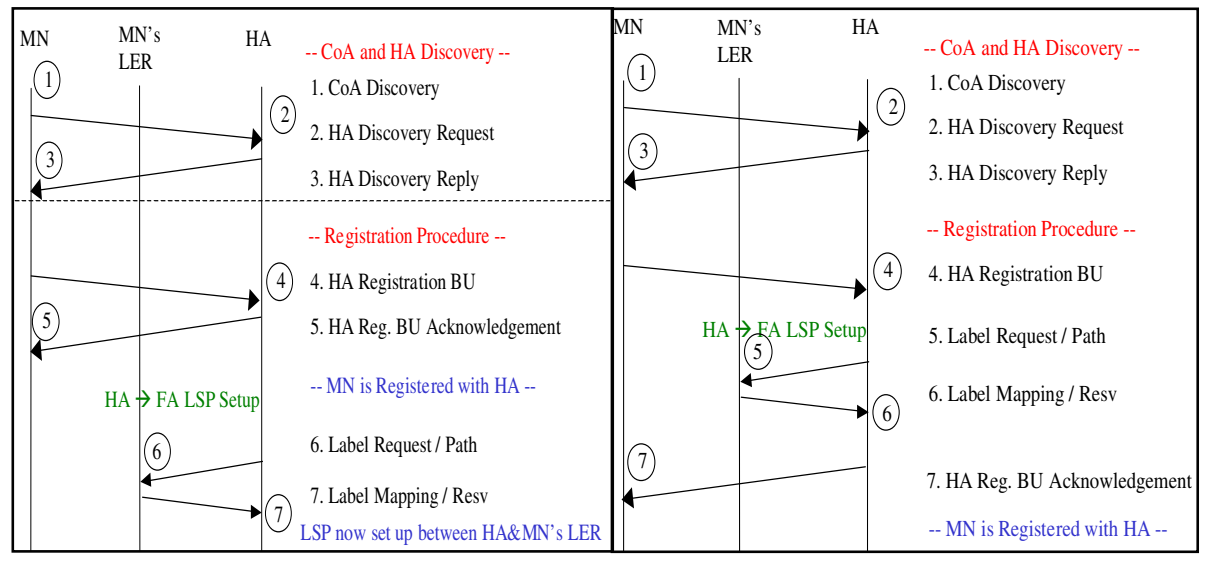

Fig. 2. HA-MA LSP setup. (a) Sequential Method and (b) Encapsulated Method.

\footnotetext{
${ }^{2}$ For a more comprehensive justification of the design points, see [9].
} 
We believe that LSPs should be set up after a binding update acknowledgement is sent back from the correspondent node, so as to avoid setting up LSPs for connections that are not going to be accepted.

Not setting the LSP before an acknowledgement is also helpful in an Integrated Services QoS environment since the other end of the connection along with the intermediate routers needs to be consulted. If we allow the mobile node to move into neighboring domains, we may want to utilize this option to make sure that any LSPs to the mobile node are set up only after the service level agreements between the different domains and the mobile node are fully negotiated and accepted. Even though we do not address them in this work, security considerations are also a factor for expecting an acknowledgement from the home agent or other nodes before proceeding.

- LSPs are set up after a binding update acknowledgement is sent back from the correspondent node.

\section{Hierarchical Mobile IP - MPLS Overlay Framework}

The overlay framework we propose is based on [3], [6], [8] and the assumptions and requirements stated in Section 2.3. In terms of architecture, we consider co-locating the mobility agents with the LSRs in the RAN. The interaction of the two is limited to the LSRs using the routing tables updated by HMIP. Procedures like HMIP registration and LSP setup are independent and databases do not share entries or reference each other.

The following subsections explain the protocol based on the specifications descrybed above. We consider two slightly different scenarios: one in which (some) IP packets are allowed to traverse the network and one in which only MPLS is used as the transport.

\subsection{Scenario 1 - MPLS Data Packets Only}

The basic signaling diagram for the scenario where only MPLS data packets are allowed is shown in Fig. 3.

\subsection{Scenario 2 - IP Data Packets Allowed}

From a closer examination of the first case in scenario 1 (Fig. 3), it seems that a lot of messages are communicated to set up a number of initial LSPs between the correspondent node, the home agent, the MAP and the mobile node's LER. All these LSPs are not used for long since the correspondent node creates a more direct path to the mobile node soon after it receives a binding update. Therefore, we consider the scenario where some IP packets are allowed to traverse the MPLS network. The information allowed to be communicated in pure IP format is limited to the packets originating from a correspondent node and directed to the home address of a mobile node when there has not been an LSP set up for such communication before. 


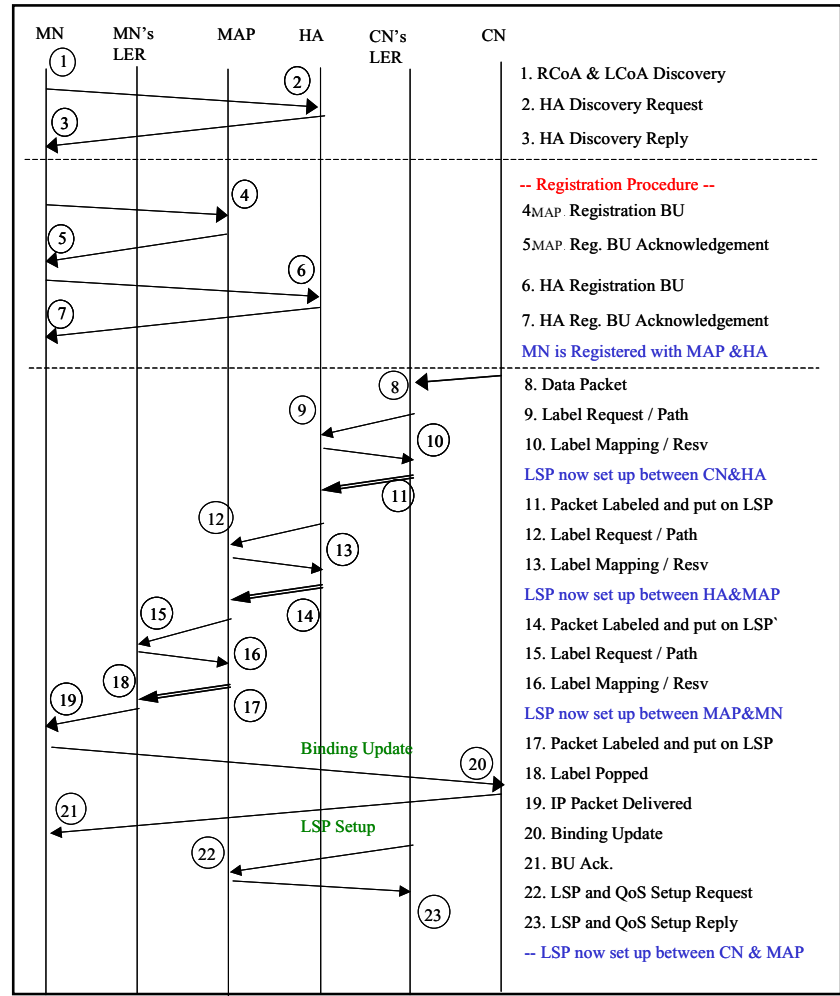

Fig. 3. HMIPv6; Data Driven HA-MA LSP; MPLS data only

\subsubsection{Correspondent Node Initiates Transmission}

There are three slightly different cases considered for this scenario:

- Case 1. CN has no binding for the MN, and CN's LER has no LSP to MN's address

- Case 2. CN has no binding for the MN, and CN's LER has LSP to MN's address

- Case 3. CN has a binding for the MN, and CN's LER has LSP to MN's new location

When a data packet arrives, or is created at the correspondent node, the node first examines its binding cache for an entry of mobile node's home address. If the correspondent node does not have an entry it sends the packet to the mobile node's home address. If MA10 does not have an LSP already established between the correspondent node and the mobile node (case 1), it proceeds by sending it using pure IP as described in the basic HMIP operation. The Regional CoA associated with the mobile node is that of MA0. When the mobile node receives a tunneled message it will send a binding update for its Regional CoA to the correspondent node, which will use it for the establishment of an LSP to the mobile node if there is a need.

This operation reduces the amount of MPLS related overhead at the initial stages of a communication. At the same time, no MPLS based QoS support is provided to those packets. DiffServ support in IP using the DSCP fields in the IP header could be used in such circumstances. In addition, if the CN-MN LSP is set up quickly, then there may be no reason for concern even if no QoS is provisioned for those packets. 
If MA10 does have an LSP set up toward the mobile node's home address (case2), it uses MPLS to send the packet. The home agent will intercept the packet after the MPLS header is stripped off and recognize that there is an entry in its binding cache for that mobile node. It will then create an encapsulated header and send the packet to the mobile node through the MAP. The rest of the operations are as in the first case.

CN's LER - MA10

\begin{tabular}{|c|c|c|c|c|r|}
\hline Input I/F & $\begin{array}{c}\text { Input } \\
\text { Label }\end{array}$ & FEC & Operation & Out I/F & $\begin{array}{r}\text { Out } \\
\text { Label }\end{array}$ \\
\hline-- & -- & MN1 & Push & 1 & 10 \\
\hline
\end{tabular}

MN's HA - MA11

\begin{tabular}{|c|c|c|c|c|c|}
\hline Input I/F & $\begin{array}{c}\text { Input } \\
\text { Label }\end{array}$ & FEC & Operation & Out I/F & $\begin{array}{r}\text { Out } \\
\text { Label }\end{array}$ \\
\hline 1 & 10 & MN1 & Pop & -- & -- \\
\hline
\end{tabular}

In the third case, the correspondent node uses the mobile node's Regional CoA from its binding cache. The CN's LER (MA10) will find the corresponding entry in the LFIB table and form MPLS packets with label 21 as the outgoing label.

CN's LER - MA10

\begin{tabular}{|c|c|c|c|c|r|}
\hline Input I/F & $\begin{array}{c}\text { Input } \\
\text { Label }\end{array}$ & FEC & Operation & Out I/F & $\begin{array}{r}\text { Out } \\
\text { Label }\end{array}$ \\
\hline-- & -- & MN1 & Push & 1 & 10 \\
\hline-- & -- & RCoA & Push & 1 & 21 \\
\hline
\end{tabular}

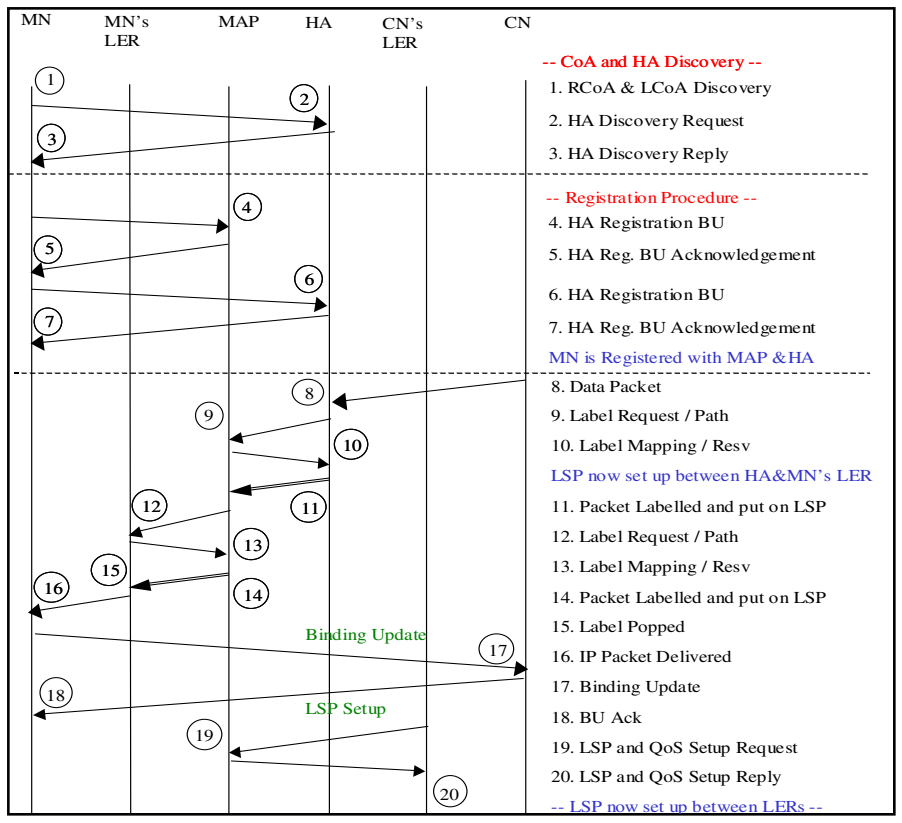

Fig. 4. HMIPv6; Data Driven HA-MA LSP establishment; IP Packets allowed 


\subsubsection{Mobile Node Initiates Communication}

In this scenario (shown in Fig. 4), some IP packets can be routed from the mobile node to the correspondent node without using MPLS. The mobile node's LER (MA5) will forward pure IP packets until it recognizes that a flow is in place.

\section{Handovers in the Overlay Framework}

When a mobile node moves out of the range of a mobility agent and into the range of another, the movement is understood by the difference in the router advertisements received. If the movement is inside the RAN (below the MAP), the handoff is an intra-RAN handoff. If the change in position is such that a new MAP is going to be used, the handoff is called inter-RAN handoff.

Regardless of the type of handoff (intra- or inter-RAN), there are many ways to perform path rerouting following a location change:

- LSP re-establishment - A new LSP created to/from the new location.

○ Advantages: simple

○ Disadvantages: Packets in transit are lost

- LSP extension - The LSP is extended from the old edge router to the new edge router

- Advantage: fast, no packets are lost

- Disadvantages: LSP length is increased, delay may be increased, loop prevention is required

- LSP extension and modification - the LSP is first extended and then modified.

- Combination of the first two methods

- Advantages: Fast, simple, no packets are lost

- LSP multicast - LSPs are created in multiple locations around the MN

- Advantages: enables fast and smooth handoff

- Disadvantages: point-to-multipoint LSPs needed, extensive location knowledge is required. Difficult to handle resources

- LSP dynamic rerouting - the LSP is modified starting from the lowest (closest to the MN) common router between the old and the new path. Partial path reestablishment.

- Improvement on extension and modification method.

- Advantages: full LSP re-establishment is not required. Parts of LSP remain the same, which means less signaling.

○ Disadvantages: increased complexity

All of the rerouting methods described above have been used in current proposals. Multicasting and dynamic rerouting seem the most popular and efficient. However, the corresponding proposals make certain assumptions on the capabilities of LDP or the level of interaction between architectures that do not fit the realities of our model of an overlay framework. Therefore, we propose the use of the LSP extension and modification method. This method is simple; it can be used without any additions to the architectures; uses existing functions (like the old MA notification and MN-CN $\mathrm{BU})$ as the basis; and also limits packet loss. 


\subsection{Intra-RAN Handoff}

Suppose that MN1 moves out of the range of MA5 to a location closer to MA6. The mobile node will obtain a new LCoA from its new location. It will then send a binding update to MA5 with the new LCoA so that packets in transit toward the MA5 are redirected toward MA6. At the same time, the mobile node will send a local binding update to its MAP (MA0) and any correspondent nodes inside the RAN. Prior to the handoff, MA5 has the following data in its LFIB:

Old LER - MA5 (before handoff)

\begin{tabular}{|c|c|c|c|c|c|}
\hline Input I/F & $\begin{array}{c}\text { Input } \\
\text { Label }\end{array}$ & FEC & Operation & Out I/F & $\begin{array}{r}\text { Out } \\
\text { Label }\end{array}$ \\
\hline 1 & 30 & LCoA1 & Pop & -- & -- \\
\hline 1 & 31 & LCoA1 & Pop & -- & -- \\
\hline-- & -- & CN & Push & 1 & 40 \\
\hline
\end{tabular}

After the handoff MA5 will initiate an LSP setup between itself and MA6. The LSP will be for LCoA2. MA5 and MA6 will update their tables accordingly.

Old LER - MA5 (after handoff)

\begin{tabular}{|c|c|c|c|c|c|}
\hline Input I/F & $\begin{array}{c}\text { Input } \\
\text { Label }\end{array}$ & FEC & Operation & Out I/F & $\begin{array}{c}\text { Out } \\
\text { Label }\end{array}$ \\
\hline 1 & 30 & LCoA1 & Pop & -- & -- \\
\hline 1 & 31 & LCoA1 & Pop & -- & -- \\
\hline-- & -- & CN & Push & 1 & 40 \\
\hline-- & -- & LCoA2 & Push & 1 & 50 \\
\hline
\end{tabular}

New LER - MA6

\begin{tabular}{|c|r|c|c|c|c|}
\hline Input I/F & $\begin{array}{c}\text { Input } \\
\text { Label }\end{array}$ & FEC & Operation & Out I/F & $\begin{array}{c}\text { Out } \\
\text { Label }\end{array}$ \\
\hline 1 & 50 & LCoA2 & Pop & -- & -- \\
\hline
\end{tabular}

The result of this extension is the aggregation of the MN LSPs at MA5 into one LSP for MA6. We do not consider this to be an important issue given that the path is only going to be used for the limited number of packets in transit (or for packets originating from MA5).

Prior to an intra-RAN handoff the MAP has an entry in its binding cache relating the RCoA and LCoA used by the mobile node, and an LSP connecting to the LER serving it. After the handoff the MAP has a different LCoA associated with the mobile node and needs to establish a path toward it. If full LSP re-establishment is used, the MAP will establish a new LSP toward the mobile node and add the entry in the LFIB. The connection between the old and the new entries is done in the binding cache. Therefore, the trend of leaving the MPLS layer in order to get the new CoA and return to find the new path to it is continued here. As with the old LER, the operation will end up aggregating all of MAP's existing LSPs to the mobile node into one, unless the MAP performs an LSP setup for every entry it has in its table. In this case, to differentiate between entries the FEC needs to contain the address of the sender node as well. Therefore in the overlay environment we also add the requirement that FECs denote end node pairs. 


\subsection{Inter-RAN handoff}

Inter-RAN handoffs include everything done in intra-RAN handoffs with the addition that the mobile node's home agent and correspondent nodes outside the RAN will have to establish LSP(s) to the mobile node's new MAP. Let us consider the case where MN1 moves into the RAN served by MA2. The home agent will receive a binding update with MA2 as the new RCoA and update its binding cache with the new value. If its connection with the mobile node is active (data present) it will also initiate an LSP setup to the new RCoA. Correspondent nodes outside the mobile node's new RAN will also have to do the same. The label forwarding information base of the HA will be changed to:

HA - MA11

\begin{tabular}{|c|c|c|c|c|c|}
\hline Input I/F & $\begin{array}{c}\text { Input } \\
\text { Label }\end{array}$ & FEC & Operation & Out I/F & $\begin{array}{c}\text { Out } \\
\text { Label }\end{array}$ \\
\hline 1 & 10 & MN1 & Pop & -- & -- \\
\hline-- & -- & RCoA1 & Push & 1 & 20 \\
\hline-- & -- & RCoA2 & Push & 1 & 60 \\
\hline
\end{tabular}

The entry for the old RCoA will remain in the table until released by the ingress or withdrawn by the egress router. There is no provision at present for the release or withdrawal of these labels based on mobile IP information.

Since MA0 will always be the correct downstream router for RCoA1 MPLS does not give the option to the upstream router to release the label. A downstream node can withdraw a label if it decides to break the binding between the label and the address prefix associated with it. The LSR withdrawing a label must do so from every LSR it has distributed that label. Label withdrawing is useful in the handoff framework only if there is a mechanism to inform MPLS that the binding is not needed anymore. This is another example where triggering MPLS events through mobile IP events is beneficial.

\section{Conclusions}

This paper proposed a framework that integrates Multi-Protocol Label Switching (MPLS) and Hierarchical Mobile IPv6 (HMIPv6) in a Radio Access Network (RAN) in a simple overlay fashion. The need for such a framework stems from the increased drive toward high-speed multimedia-intensive services.

The overlay method proposed improves on existing methods of MIP-MPLS interworking based on MIPv4 and HMIPv4 and utilizes HMIPv6 as the micromobility protocol.

Detailed operation signaling diagrams as well as forwarding table contents were presented and the ability of the protocol to handle mobility events (handover) was illustrated both for the intra and inter-RAN cases.

In conclusion, we find that MPLS, when paired with a suitable mobility protocol can function well in a radio access network and provide the same benefits it offers when used in wired networks. 


\section{Acknowledgements}

This work has been partly supported by the European Union under the project E-NEXT FP6506869.

\section{References}

[1] E. Rosen, A. Viswanathan and R. Callon, "Multiprotocol Label Switching Architecture," Request for Comment 3031, Internet Engineering Task Force, Jan. 2001.

[2] C. Perkins Ed., "IP Mobility Support", Request for Comment 3220, Internet Engineering Task Force, Jan. 2002.

[3] H. Soliman, C. Castelluccia, K. El-Makri, and L. Bellier, "Hierarchical Mobile IPv6 Mobility Management (HMIPv6)," Request for Comment 4140, Internet Engineering Task Force, Aug. 2005.

[4] C. Perkins, D. Johnson, and J. Arkko "Mobility Support in IPv6," Request for Comment 3775, Internet Engineering Task Force, Jun. 2004.

[5] V. Vassiliou, H. L. Owen, D. A. Barlow, J Grimminger, H-P Huth, and J. Sokol, "A Radio Access Network for Next Generation Wireless Networks Based on MPLS and Hierarchical Mobile IP,” In Proc. IEEE 56th Vehicular Technology Conference Fall 2002 (VTC2002Fall), Vancouver, Canada,. pp 782-786, Sep. 2002.

[6] V. Vassiliou, H. L. Owen, D. A. Barlow, J Grimminger, H-P Huth, and J. Sokol, "MMPLS: Micromobility-enabled Multiprotocol Label Switching," In Proc. IEEE International Conference on Communications (ICC2003), Alaska, USA, May 2003.

[7] Barlow, D., Vassiliou, V., Krasser, S., Owen, H., Grimminger, J., Huth, H.-P., Sokol, J., "Traffic Engineering Based on Local States In Internet Protocol-based Radio Access Networks", Journal Computer Networks, Vol. 7, No. 3, September 2005, pp. 377-384.

[8] J. K. Choi, M.H. Kim, and T.W. Um, "Mobile IPv6 support in MPLS," Internet Draft <draft-choi-mobileip-ipv6-mpls-01.txt>, Aug. 2001.

[9] V. Vassiliou, "An Integration Framework and a Signaling Protocol for MPLS/ DiffServ/ HMIP Radio Access Networks,” Ph.D. Thesis, Georgia Institute of Technology, Jul. 2002. 